\title{
Wind Models for O-type Stars
}

\author{
T.L. Hoffmann, A.W.A. Pauldrach \\ Institut für Astronomie und Astrophysik der Universität München, \\ Scheinerstraße 1, 81679 München, Germany
}

\begin{abstract}
We present fully consistent models for O-type stars that reproduce the observed UV spectra simultaneously with the observed terminal velocities and mass loss rates. We demonstrate how, as a consequence, the models can be used to determine the stellar parameters from the observed UV spectra alone.
\end{abstract}

\section{Comparison with observations}

For a detailed description of the treatment of non-LTE, radiation transfer, and line blocking and blanketing in our models we refer to Pauldrach et al. (2001). Here we focus on the aspect of consistent hydrodynamics which we have recently incorporated into our procedure (see Pauldrach and Hoffmann 2002), and show that these models can well reproduce all main features of the observed winds of individual massive $\mathrm{O}$ stars.

To test the quality of the models we have selected a sub-sample with wellknown parameters and a wide temperature range from the sample of stars analyzed by Puls et al. (1996), for which we have computed models with hydrodynamics and NLTE iterated to consistency. (The hydrodynamics affects the NLTE model via density structure and velocity field (radiative transfer with Doppler-shifted spectral lines), but in turn is controlled by the line force determined by the occupation numbers and radiative transfer of the NLTE model.)

For the same stellar parameters $\left(T_{\text {eff }}, \log g, R\right)$ our models give terminal velocities $\left(v_{\infty}\right)$ within $10 \%$ of the observed values and mass loss rates $(\dot{M})$ within a factor of 2 of those determined by Puls et al., as shown in the figures below. Since the analysis by Puls et al. did not yet include line blanketing, we expect the remaining small discrepancies to be further reduced by the currently ongoing reanalysis of the sample with an up-to-date method.
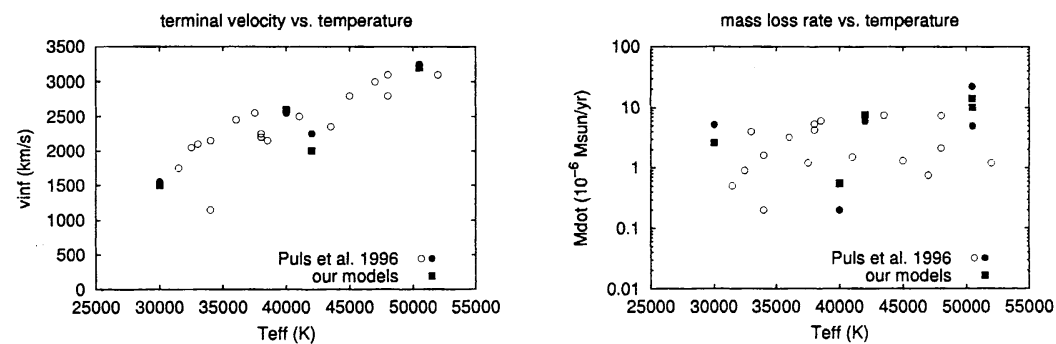


\section{Determining stellar parameters from the UV spectrum}

Consistent hydrodynamics provides the link between the stellar parameters $\left(T_{\text {eff }}\right.$, $\log g, R)$ and the wind parameters $\left(v_{\infty}, \dot{M}\right)$; it is the interplay of the NLTE model and the hydrodynamics that determines the appearance of the UV spectrum. Computing the wind dynamics consistently permits not only the determination of the wind parameters from given stellar parameters, but conversely makes it possible to obtain the stellar parameters from the observed UV spectrum alone. Although this idea is not new (see Pauldrach et al. 1988, Kudritzki et al. 1992), only the recent generation of models has reached the degree of sophistication that makes such a procedure practicable instead of purely academic. An application of this method to O-type central stars of planetary nebulae is given by Pauldrach et al. (these proceedings).

To briefly illustrate the effect of a change in radius and gravity on the spectra and wind parameters, we have calculated a grid of models with $T_{\text {eff }}=$ $40000 \mathrm{~K}$ and consistent wind dynamics, using radii from 15 to $25 \mathrm{~L}_{\odot}$ and $\log g$ from 3.4 to 4.0 The resulting mass loss rates and terminal velocities as well as the corresponding UV spectra are shown below. In principle, one can now immediately read off the stellar parameters simply by comparing an observed UV spectrum to such a grid at the appropriate effective temperature. (In the grid of spectra below, $R$ increases to the right, $\log g$ increases toward the bottom.)
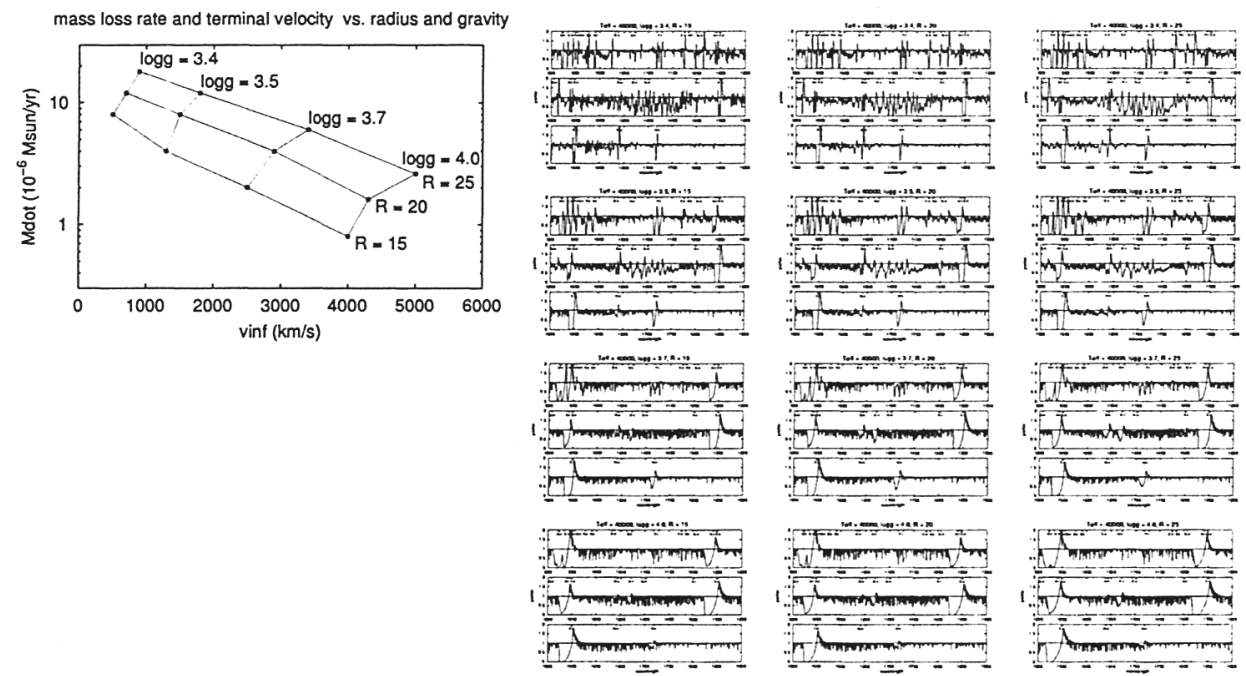

Acknowledgments. This work was supported by the DLR under grant 50 OR 99092.

\section{References}

Kudritzki R.-P., Hummer D. G., Pauldrach A. W. A., et al. 1992, A\&A 257, 655

Pauldrach A. W. A., Puls J., Kudritzki R.-P., et al. 1988, A\&A 207, 123

Pauldrach A. W. A., Hoffmann T. L., Méndez R. H., these proceedings

Pauldrach A. W. A., Hoffmann T. L., Lennon M., 2001 A\&A 375, 161

Pauldrach A. W. A. \& Hoffmann T. L., 2002 A\&A, 375, 161

Puls J., Kudritzki R.-P., Herrero A., et al., 1996 A\&A 307, 171 